\title{
Images in Anesthesia: Intrathoracic leiomyosarcoma in a parturient presenting for Cesarean delivery
}

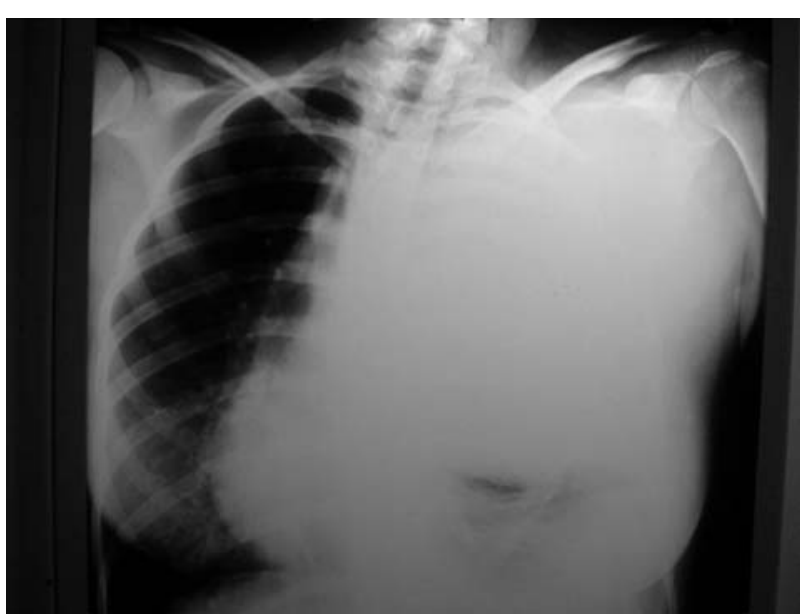

FIGURE lA Chest $x$-ray.

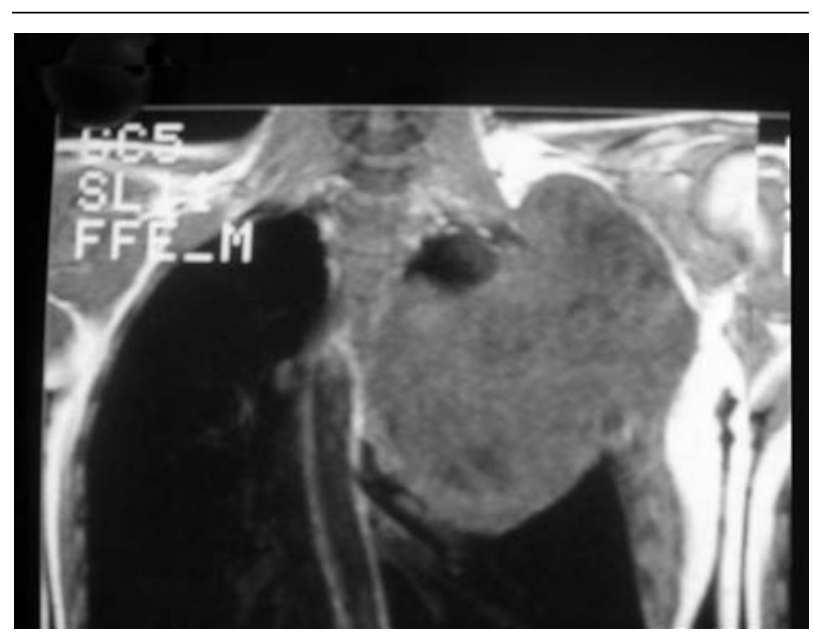

FIGURE IB Magnetic resonance imaging demonstrating large left-sided intrathoracic tumour.

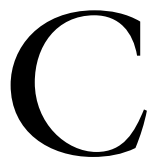

ROSBY first reported on the anesthetic management (spinal block) of a parturient with a large intrathoracic tumour (Ewingtype sarcoma) presenting for Cesarean section. ${ }^{1}$ We present a similar case, and the first report of an intrathoracic leiomyosarcoma in pregnancy. A 21 -yrold, previously healthy female at 30 weeks gestation presented with increasing fatigue and a one-week history of dyspnea, cough and hemoptysis. At admission she was hemodynamically stable and afebrile. A chest $x$-ray demonstrated an intrathoracic lesion in the left hemithorax (Figure 1A). Magnetic resonance imaging studies confirmed a large tumour in the left hemithorax (caphalo-caudal, transverse and antero-posterior diameters were $155 \mathrm{~mm}, 176 \mathrm{~mm}$, and $136 \mathrm{~mm}$, respectively), invading the left pleural cavity, and left axilla (Figure 1B). A fine needle biopsy yielded the diagnosis of leiomyosarcoma. Uneventful Cesarean delivery was performed under single dose spinal anesthesia with 12.5 $\mathrm{mg}$ of bupivacaine. Postpartum the patient received chemotherapy, which proved futile and she expired (cardiorespiratory failure) three weeks after delivery. Leiomyosarcoma is an extremely rare neoplasm. ${ }^{2}$ Due to its rarity and non-specific clinical symptoms, the diagnosis and treatment are often delayed. In pregnan$\mathrm{cy}$, the physiological changes of pregnancy may mask not only the initial presentation but also the advanced stage of the neoplasm.

Claudia L. Fernandez MD*

Krzysztof M. Kuczkowski MD†

Buenos Aires, Argentina*

San Diego, California†

\section{References}

1 Crosby E. Clinical case discussion: anesthesia for Cesarean section in a parturient with a large intrathoracic tumour. Can J Anesth 2001; 48: 575-83.

2 Kagami S, Kashimura M, Toki N, Katuhata $\Upsilon$. Myxoid leiomyosarcoma of the uterus with subsequent pregnancy and delivery. Gynecol Oncol 2002; 85: 538-42. 